\title{
A RARE BIFURCATION PATTERN OF THE SCIATIC NERVE
}

\author{
Emranul Huq ${ }^{1}$, Paul Bailie ${ }^{2}$ \\ ${ }^{1}$ Assistant Professor (Anatomy), Faculty of Basic Sciences, Saint James School of Medicine - St. Vincent and the \\ Grenadines campus. \\ ${ }^{2}$ MD Candidate, Saint James School of Medicine - Anguilla campus.
}

Correspondence to Prof. Emranul Huq, Saint James School of Medicine. PO Box 2336. Cane Hall, St. Vincent and the Grenadines. Phone: +1784-496-0236. Email: ehuq@mail.sjsm.org.

\begin{abstract}
Variations in branching patterns of the sciatic nerve are thought to be clinically significant because of the nerve's extensive distribution area. Here we report a rare and unusual branching pattern of the sciatic nerve which was observed in a male cadaver. Sciatic nerve underwent a high division inside the pelvic cavity, and entered the gluteal region as separate tibial and common fibular nerves. Subsequent distal courses of both nerves into the leg were normal. Knowledge of sciatic nerve variations is useful in treating lower limb neuropathies, such as piriformis syndrome, which tends to be caused by the compression of the sciatic nerve by the piriformis muscle.
\end{abstract}

Keywords: Sciatic nerve bifurcation; sciatic nerve anomalies; piriformis syndrome.

\section{INTRODUCTION}

The sciatic nerve is the largest nerve in the human body (Williams et al., 1995). It is one of the terminal nerves emerging from the lumbosacral plexus, and is formed by the union of the ventral rami of L4-S3 spinal nerves (Williams et al., 1995; Fessler and Sekhar, 2006). The sciatic nerve tends to exit the pelvic cavity through the greater sciatic notch below the piriformis, and continues distally along the posterior compartment of the thigh. Once the nerve reaches the apex of the popliteal fossa, it bifurcates into tibial and common fibular nerves, which continue distally and innervate structures in the leg and the foot (Williams et al., 1995).

Even though the sciatic nerve is typically present as a single nerve until it reaches the popliteal fossa, the two divisions of the sciatic nerve occasionally separate from one another proximal to the apex of the popliteal fossa (Beaton and Anson, 1937; Williams et al., 1995; Fessler and Sekhar, 2006). The objective of this report is to describe a relatively rare branching pattern of the sciatic nerve observed in one male cadaver during routine anatomical dissection. The significance of documenting anatomical variations of the sciatic nerve is to facilitate effective treatment of sciatic nerve neuropathies, as well as to avoid iatrogenic injuries and encourage the use of proper imaging techniques for identifying anomalies (Paval and Nayak, 2006; Saleh et al., 2009; Saritha et al., 2012; Russa and Fabian, 2017).

\section{CASE REPORT}

During a routine dissection session for medical students at the Saint James School of Medicine - Anguilla, skin, fat, and fasciae were removed from both lower limbs of a male cadaver; and muscles and neurovascular structures were dissected and defined. 
In the course of dissection, an anomaly was noticed in the right lower limb. Two nerves, as opposed to a single sciatic nerve, were observed along the entire length of the posterior thigh (Figure 1). The nerves were followed proximally into the gluteal region, where the common fibular nerve was seen to be emerging inferior to the piriformis muscle (Figure 1 ), which is the typical anatomical location for the exit of the sciatic nerve from the pelvic cavity (Williams et al., 1995). The tibial nerve, on the other hand, emerged inferior to the superior gemellus muscle (Figure 1). When the nerves were traced farther proximally into the pelvic cavity, it was observed that the ventral rami of spinal nerves L4-S3 had combined to form the sciatic nerve with an approximate length of $3 \mathrm{~cm}$ (Figure 2), which had subsequently divided into tibial and common fibular branches, and entered the gluteal region as such (instead of as a single sciatic nerve) (Figure 1).

When the tibial and common fibular nerves were followed distally along the posterior compartment of the right thigh, the latter was seen to enter the leg by passing deep to the tendon of the biceps femoris muscle, while the former entered the leg between the two heads of the gastrocnemius muscle (Figure 1). Thus, distal courses of both nerves appeared to have followed their standard patterns (Williams et al., 1995).

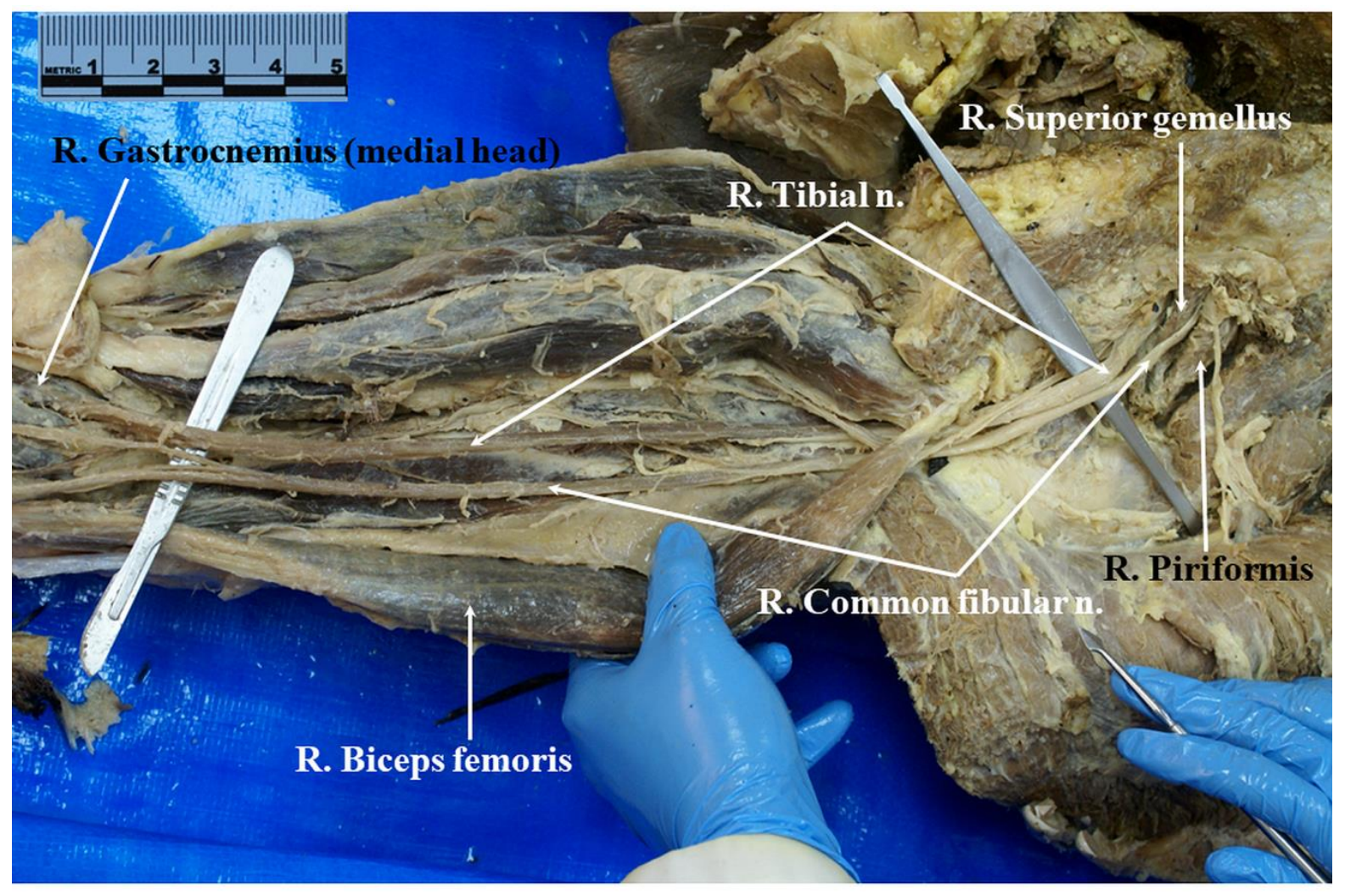

Figure 1: Courses of tibial and common fibular nerves in posterior compartment of right thigh 


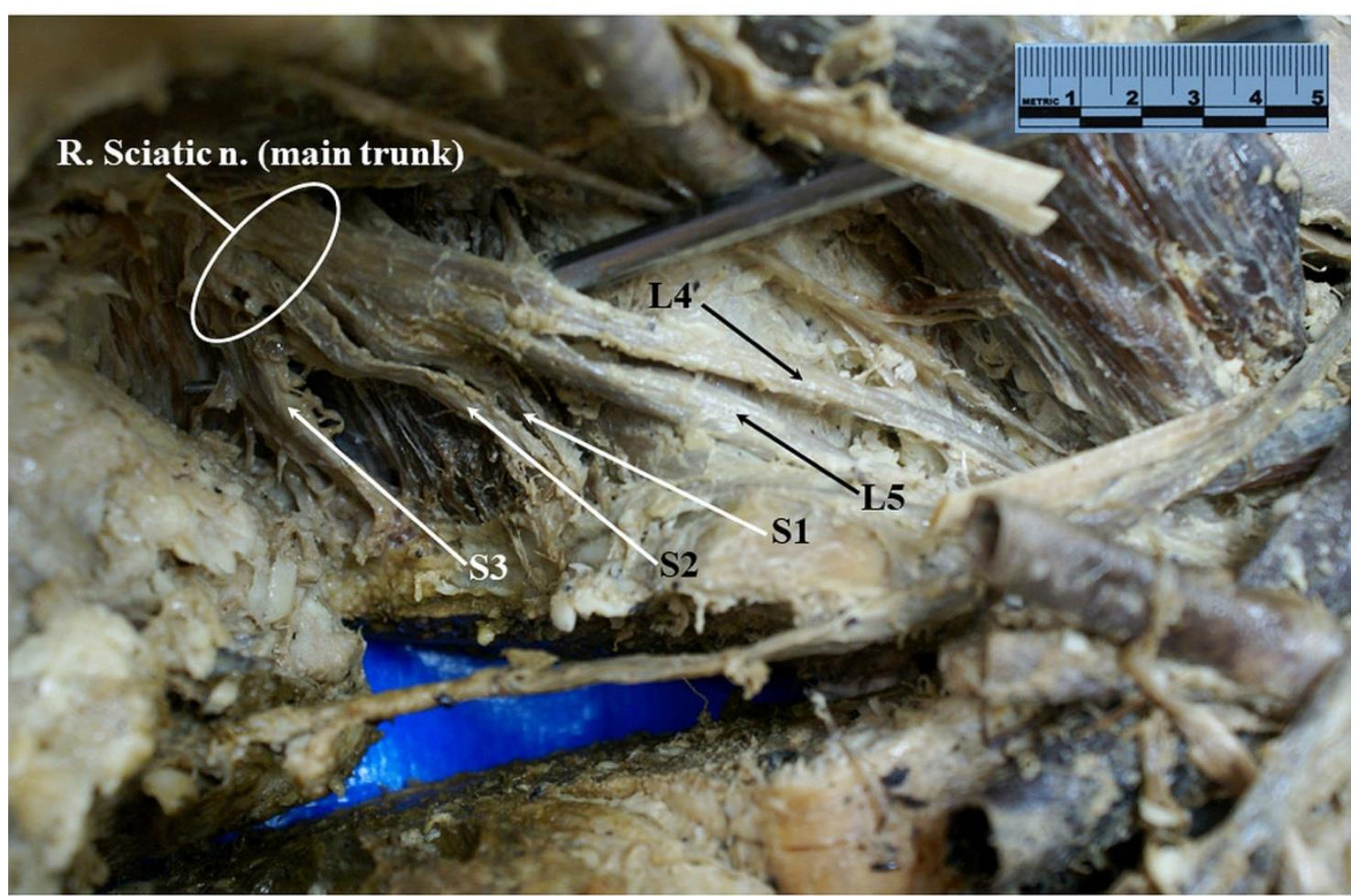

Figure 2: Formation of the main trunk of the right sciatic nerve by ventral rami of L4-S3 spinal nerves.

\section{DISCUSSION}

The current presentation of the anomalous branching pattern of the sciatic nerve is not included in Beaton and Anson's (1937) standard classification of sciatic nerve anomalies. A survey of the available literature shows a number of cases of sciatic nerve bifurcation pattern similar to the one reported here (e.g., Babinski et al., 2003; Mas et al., 2003; Paval and Nayak, 2006; Ogeng'o et al., 2011). Since the sciatic nerve itself had been observed inside the pelvic cavity, it appears that the current case may be best described as that of an unusual length-reduction and high division of the nerve. The importance of documentation of such anomalies is to advance the scientific knowledge and understanding of anatomical structures and their functions. For example, piriformis syndrome is a neuropathy which is believed to be caused by an irritation of the sciatic nerve due to spastic contraction, hypertrophy, or atrophy and tightness of the piriformis muscle, or secondary adhesion of the sciatic nerve to the piriformis muscle and surrounding tissue (Stewart, 1993; Filler et al., 2005). Individuals with piriformis syndrome typically present with buttock pain that may or may not radiate along the posterior thigh (Stewart, 1993); although numbness or muscle weakness is rarely reported (Filler et al., 2005). It may be speculated that in case of piriformis syndrome in individuals with the particular anomaly of the sciatic nerve reported here (emergence of the common fibular neve inferior to the piriformis), patients would be more likely to experience pain in the distribution areas of the common fibular nerve, e.g., anterior and lateral compartments of the leg and dorsal compartment of the foot (Fessler and Sekhar, 2006), as opposed to along the entire posterior compartment of the lower limb. Similarly, an entrapment or compression of the tibial nerve by the superior gemellus muscle (as reported here) would be expected to produce pain in the distribution areas of the tibial nerve. 
A number of investigators suggest that the probability of the presence of at least one anomaly of the piriformis muscle and sciatic nerve on one or both sides is approximately 23\% (Broadhurst et al., 2004; Smoll, 2010). However, Smoll (2010) also notes that explicit connection between piriformis and sciatic nerve anomalies to the piriformis syndrome has been rarely observed; nor does there seem to be any consistent relationship among these factors (Broadhurst et al., 2004).
Therefore, it is possible that the roles of piriformis and sciatic nerve anomalies in producing piriformis syndrome may have been overstated (Broadhurst et al., 2004; Smoll, 2010). Nevertheless, while more research is needed to establish proper relationship(s) between sciatic nerve variations and piriformis syndrome, a sound knowledge of sciatic nerve anomalies would be important for proper diagnosis and management of the piriformis syndrome (Filler et al., 2005).

Conflict of interest: The authors declare that there is no conflict of interest regarding the publication of this paper.

Acknowledgements: The authors wish to thank Mr. Moses Loli for his help with dissection and photography.

\section{REFERENCES}

1. Babinski MA, Machado FA, Costa WS. 2003. A rare variation in the high division of the sciatic nerve surrounding the superior gemellus muscle. Eur J Morphol. 41: 41-42.

2. Beaton LE, Anson BJ. 1937. The relation of the sciatic nerve and of its subdivisions to the piriformis muscle. Anat Rec. 70: 1-5.

3. Broadhurst NA, Simmons DN, Bond MJ. 2004. Piriformis syndrome: correlation of muscle morphology with symptoms and signs. Arch Phys Med Rehabil. 85: 2036-2039.

4. Fessler RG, Sekhar LN. 2006. Atlas of neurosurgical techniques: spine and peripheral nerves. $1^{\text {st }}$ ed. New York: Thieme; pp. 1-1048.

5. Filler AG, Haynes J, Jordan SE, Prager J, Villablanca JP, Farahani K, et al. 2005. Sciatica of nondisc origin and piriformis syndrome: diagnosis by magnetic resonance neurography and interventional magnetic resonance imaging with outcome study of resulting treatment. ] Neurosurg Spine. 2: 99-115.

6. Mas N, Ozeksi P, Ozdemir B, Kapakin S, Sargon MF, Celik HH, et al. 2003. A case of bilateral high division of the sciatic nerves, together with a unilateral unusual course of the tibial nerve. Neuroanatomy. 2: 13-15.

7. Ogeng'o JA, El-Busaidy H, Mwika PM, Khanbhai MM, Munguti J. 2011. Variant anatomy of sciatic nerve in a black Kenyan population. Folia Morphol (Warsz). 70: 175-179.

8. Paval J, Nayak S. 2006. A case of bilateral high division of sciatic nerve with a variant inferior gluteal nerve. Neuroanatomy. 5: 33-34.

9. Russa AD, Fabian FM. 2017. A unique quadrification of the sciatic nerve in the lower leg. Anat J Africa. 6: 919-923.

10. Saleh HAM, El-fark MMO, Abdel-Hamid GA. 2009. Anatomical variation of sciatic nerve division in the popliteal fossa and its implication in popliteal nerve blockade. Folia Morphol (Warsz). 68: 256-259.

11. Saritha S, Praveen KM, Supriya G. 2012. Anatomical variations in the bifurcation of the sciatic nerve, a cadaveric study and its clinical implications. Anat Physiol. 2: 111. DOI: 10.4172/2161-0940.1000111.

12. Smoll NR. 2010. Variations of the piriformis and sciatic nerve with clinical consequence: a review. Clin Anat. 23: 8-17.

13. Stewart JD. 1993. Focal peripheral neuropathies. $2^{\text {nd }}$ ed. New York: Raven Press; pp. 1-506.

14. Williams PL, Bannister LH, Gray H. 1995. Gray's anatomy. 38 ${ }^{\text {th }}$ ed. London: Churchill Livingstone; pp. 1-2092. 